\section{The suppression model remains unsound: A reply to Deutsch}

CHRISTOPHER D. CHAMBERS and

JASON B. MATTINGLEY

University of Melbourne, Melbourne, Victoria, Australia

and

\author{
SIMON A. MOSS \\ Monash University, Clayton, Victoria, Australia
}

In the present issue, we critically review the influential suppression model of the octave illusion (Chambers, Mattingley, \& Moss, 2004). In a rejoinder to our article, Deutsch (2004b) defends the utility of the suppression model. Here, we respond to Deutsch's arguments, many of which are based on criticisms of our recent experimental report (Chambers, Mattingley, \& Moss, 2002). We argue that Deutsch's criticisms of this previous investigation are unfounded and that her defense of the suppression model fails to account for the most important weaknesses of this theory. We conclude that the suppression model remains inadequate as an explanation of the octave illusion.

The octave illusion presents a significant challenge to our understanding of auditory perception. In the present issue, we raised concerns about the popular explanation of the octave illusion, termed the suppression model (Chambers, Mattingley, \& Moss, 2004). In a response to our article, Deutsch (2004b) challenges our critique of the suppression model. ${ }^{1}$ Here, we reply to Deutsch's (2004b) arguments, most of which are based on criticisms of our previous experimental report (Chambers, Mattingley, \& Moss, 2002). In our view, no aspect of Deutsch's (2004b) response changes the conclusion reached from the available evidence: The suppression model remains inadequate.

\section{Deutsch's Criticisms of the Experiments Conducted by Chambers et al. (2002)}

Deutsch (2004b) raises several objections to our experimental work on the octave illusion. Before addressing Deutsch's (2004b) defense of the suppression model, we reply to these criticisms of our previous study (Chambers et al., 2002).

The influence of tone duration and interonset interval on the octave illusion. Deutsch (2004b) questions our observation that subjective reports of the octave illusion were unchanged by tone duration and the inclusion of silent intervals between tones (Chambers et al., 2002). The author describes research by Zwicker (1984) that

Correspondence concerning this article should be addressed to C. D. Chambers, Cognitive Neuroscience Laboratory, Department of Psychology, University of Melbourne, Victoria 3010, Australia (e-mail: c.chambers@psych.unimelb.edu.au). showed an effect of tone duration on the percept obtained through subjective report. Deutsch (2004b) concludes that "the temporal parameters employed by Chambers et al. (2002) included some that, on the basis of Zwicker's findings, should often have resulted in no illusion" (p. 670).

We agree that some studies have shown an influence of temporal parameters on subjective reports of the octave illusion. However, Deutsch's (2004b) argument ignores the variability of findings on this issue. Consider, for instance, the article by Deutsch (1980a) that is reviewed in section 4.2 of Chambers et al. (2004). ${ }^{2}$ In Experiment 3, Deutsch (1980a) elicited the octave illusion, using sequences with an interonset interval of $1 \mathrm{sec}$. This finding is consistent with the subjective reports obtained in Chambers et al. (2002) and differs from those obtained by Zwicker (1984). ${ }^{3}$ In our view, Deutsch (2004b) overstates the implications of findings that demonstrate a relationship between interonset interval and subjective reports of the illusion. Subjective reports are susceptible to a range of distortions, including response bias and decision noise. Such reports are useful only as a supplement to objective psychophysical experiments. We suggest that firm conclusions regarding the effects of temporal parameters on the octave illusion will require systematic investigations in which objective techniques are used.

The influence of prior exposure and naiveté on the octave illusion. Deutsch (2004b) claims that prior exposure to single dichotic octaves might have altered the percept of the illusion obtained in Chambers et al. (2002). The author also argues that, for nonnaive listeners, "knowledge of the stimulus pattern could have affected their judgments" (p. 670). However, Deutsch (2004b) presents no evidence to suggest that prior exposure or naiveté to dichotic stimuli influences the illusion. Furthermore, it is noteworthy that Deutsch has served as a listener in her own published studies of the octave illusion (e.g., Deutsch, 1980a). We know of no evidence that would invalidate the inclusion of such nonnaive subjects.

The magnitude of pitch variations reported in Chambers et al. (2002). Deutsch (2004b) suggests that many of the reported pitch variations in Experiment 1 of Chambers et al. (2002) were too large to be explained by diplacusis. This argument, however, overestimates the precision of these magnitude estimates, which were based on subjective reports. The critical aspect of these findings was that of the 13 listeners who reported a pitch variation during the octave illusion, 11 reported a suboctave alternation. This initial result raised doubts about the suppression explanation that were confirmed in subsequent psychophysical experiments.

The tapping task used in Experiment 1 of Chambers et al. (2002). Deutsch (2004b) questions the validity of the tapping task used in Chambers et al. (2002) to assess the pitch and lateralization percepts of the octave 
illusion. The author queries how the tapping was synchronized with the stimulus presentation to ensure correct frequency-location assignments by the experimenter. We used visual cues to ensure that this measurement was performed accurately. The cues were presented synchronously with the onset of each stimulus and occurred at different locations for each configuration of the dichotic octaves. To determine which dichotic octave was localized to which side, the experimenter monitored the display to see which visual cue was in synchrony with the tapping. The experimenter and subjects performed this task easily. As was stated in Chambers et al. (2002), listeners were instructed to tap in time with either the pitch or the lateralization percept (e.g., tap in time with the higher pitch; tap in time with the left tone). At the fastest rate of the illusion (200 $\mathrm{msec}$, no silent interval), listeners thus tapped once every $400 \mathrm{msec}$. Healthy subjects find this rate of tapping comfortable and achievable; studies in which tapping has been examined more formally have used comparable rates (e.g., Repp, 2001).

Deutsch's criticism of Experiment 3 in Chambers et al. (2002). As is described in Chambers et al. (2004, section 4.3), we employed a frequency segregation task to examine the role of sequential interactions in the octave illusion. Contrary to the predictions of the suppression model, alternating sequential presentation did not influence the ability of listeners to perceptually segregate the low- and the high-frequency components of a dichotic octave.

Deutsch (2004b) raises two objections to this experiment. First, she claims that exposure to octave illusion sequences in Experiment 1 may have generated veridical percepts in Experiment 3. However, this possibility is highly unlikely. Listeners never received feedback in either Experiment 1 or Experiment 3, so there were never any cues available for them to infer the signals being presented; furthermore, a 9-month period elapsed between the two experiments. Most important, however, Deutsch's (2004b) criticism does not address the central finding of Experiment 3 that segregation performance was unaffected by sequential presentation. This critical result is inconsistent with the predictions of the suppression model.

More generally, Deutsch (2004b) also claims that Experiment 3 in Chambers et al. (2002) did not address perception of the octave illusion, because listeners judged the stimuli rather than the percept. This argument does not appreciate that all the predictions in Experiment 3 followed directly from the perception of the octave illusion. The bulk of perception research utilizes objective measures, including detection, discrimination, identification, and localization tasks. Deutsch (2004b) argues that Experiment 3 did not measure perception because the task was objective rather than subjective. Considered against a long history of objective psychophysics, this claim is untenable.

Deutsch's criticism of Experiment 4 in Chambers et al. (2002). As is outlined in section 1 of Chambers et al. (2004), we examined the perception of the octave illusion in Experiment 4 by using a discrimination task. ${ }^{4}$ The results indicated that the pitch percept of the illusion always approximates the fundamental frequency, which is consistent with a fusion explanation of the illusion but is inconsistent with the suppression model.

Deutsch (2004b) argues that our conclusion from Experiment 4 of Chambers et al. (2002) is invalid because (1) "the way the octave illusion is perceived varies depending on sequential context" (p. 670), and because (2) "the deviant tones were presented simultaneously to both ears" (p. 670). However, she provides no further rationale for either of these claims. In Experiment 4, each trial ended upon detection of the deviant stimulus. To suggest that this task altered the illusion would imply that the deviant altered perception before it was presented. Similarly, Deutsch (2004b) presents no logical or evidentiary rationale for her claim that the binaural presentation of deviants in Experiment 4 invalidates the interpretation.

\section{Deutsch's Defense of the Suppression Model}

Having addressed Deutsch's (2004b) criticisms of our previous research, we now consider her defense of the suppression model.

Octave pitch variations. Deutsch (2004b) claims that most listeners perceive an octave pitch variation during the illusion, contrary to previous findings (Chambers et al., 2002; Huang, Pastore, \& Hall, 1992). In support of this argument, Deutsch (2004b) cites two previous studies of the octave illusion and a recent replication (Deutsch, 1974, 2004a; Zwicker, 1984). We agree that octave pitch variations between the low- and the highfrequency components of the illusion constitute the most convincing evidence for a suppression mechanism. However, we also note the potential ambiguity between virtual pitches that may arise from two-tone presentation (Chambers et al., 2004, section 6.1.2; Terhardt, 1974). Such octave confusions between virtual pitches could elicit an octave pitch difference without requiring suppression. Further experimentation using objective psychophysics is necessary (1) to verify the observation that some listeners reliably perceive an octave pitch variation between the low- and the high-frequency components of the illusion and (2) to determine whether such listeners process the illusion differently from those who report smaller pitch variations. At present, there is no objective evidence to support either contention. Instead, our research has suggested that the pitch percept during the octave illusion approximates the fundamental frequency (Chambers et al., 2002).

Subjective reports and response bias. As is outlined in Chambers et al. (2004, section 4), a major limitation of previous research on the octave illusion has been a reliance on subjective measures of perception. In many cases, these paradigms assess the predictions of the suppression account by measuring the consistency of subjective reports under various conditions (e.g., Deutsch, 
1978, 1980a). However, the fundamental inseparability of these "consistency" measures from response bias presents a serious interpretative weakness.

Deutsch (2004b) disagrees with our assertion that the suppression model is weakened by a dependence on subjective evidence. Specially, she argues that the counterbalanced design of the studies described in Deutsch (1978, 1980a, 1980b) controls for response bias. ${ }^{5}$ This claim is incorrect. The various counterbalancing procedures that Deutsch (2004b) describes are orthogonal to the fundamental correlation between the pitch percept and the lateralization percept in the octave illusion. This correlation presents an interpretative problem for subjective measures of the illusion because it enables subjects to maintain a consistent pattern of responses when the percept in one dimension (e.g., pitch) is uncertain.

Spectral dominance in the octave illusion. As is described in Chambers et al. (2004, section 2), the suppression model is inconsistent with theories of related illusions. We illustrated these discrepancies by comparing the octave illusion with binaural interference, the precedence effect, and the Franssen effect.

As Deutsch (2004b) points out, these illusions arise from signals different from those that produce the octave illusion. Because of these differences, she claims that, "one cannot appropriately compare these ... phenomena here." (p. 675). In an earlier discussion, however, Deutsch (1980b) speculated that the octave illusion "falls into the class of mechanisms (such as those underlying the precedence effect) that operate to counteract misleading effects of echoes and reverberation" (p. 586). We agree with Deutsch (1980b) that aspects of these illusions may be explained by auditory mechanisms that serve related functions. Thus, the relationship between the octave illusion and other localization phenomena is of critical importance in assessing the validity of the suppression model.

Electrophysiological studies. Deutsch (2004b) claims that the results of Ross, Tervaniemi, and Näätänen's (1996) electrophysiological study of the octave illusion are "entirely consistent with the two-channel model of the illusion" (p. 675). However, Deutsch provides no indication as to which aspect of their results is consistent with the suppression account. We reiterate our argument that Ross et al.'s findings can be interpreted as a failure of the suppression model. Recent psychophysical evidence presented by Sonnadara and Trainor (2004) is consistent with this contention.

\section{Summary and Conclusions}

In this article, we have replied to Deutsch's (2004b) claim that the suppression model of the octave illusion remains viable. Deutsch's (2004b) arguments are based primarily on purported shortcomings of our previous experimental report (Chambers et al., 2002), and we have shown that her criticisms of this study are unjustified. Furthermore, Deutsch's (2004b) defense of the suppression model fails to account for key weaknesses in studies claiming to provide evidence for suppression. We conclude that the suppression model of the octave illusion is fundamentally flawed, and will remain so until objective psychophysical evidence is presented to suggest otherwise.

\section{REFERENCES}

Akerboom, S., ten Hoopen, G., \& VAn der Knoop, A. (1985). Does the octave illusion evoke the interaural tempo illusion? Perception \& Psychophysics, 38, 281-285.

Chambers, C. D., MatTingley, J. B., \& Moss, S. A. (2002). The octave illusion revisited: Suppression or fusion between ears? Journal of Experimental Psychology: Human Perception \& Performance, 28, 1288-1302.

Chambers, C. D., Mattingley, J. B., \& Moss, S. A. (2004). Reconsidering evidence for the suppression model of the octave illusion. Psychonomic Bulletin \& Review, 11, 642-666.

DeuTSCH, D. (1974). An auditory illusion. Nature, 251, 307-309.

Deutsch, D. (1978). Lateralization by frequency for repeating sequences of dichotic 400- and $800-\mathrm{Hz}$ tones. Journal of the Acoustical Society of America, 63, 184-186.

Deutsch, D. (1980a). Ear dominance and sequential interactions. Journal of the Acoustical Society of America, 67, 220-228.

DEuTsch, D. (1980b). The octave illusion and the what-where connection. In R. S. Nickerson (Ed.), Attention and performance VIII (pp. 575-592). Hillsdale, NJ: Erlbaum.

Deutsch, D. (2004a). The octave illusion revisited again. Journal of Experimental Psychology: Human Perception \& Performance, $\underline{\mathbf{3 0}}_{2}$ 355-364.

DEUTsCH, D. (2004b). Reply to "Reconsidering evidence for the suppression model of the octave illusion," by C. D. Chambers, J. B. Mattingley, and S. A. Moss. Psychonomic Bulletin \& Review, 11, 667-676.

Deutsch, D., \& Roll, P. L. (1976). Separate "what" and "where" decision mechanisms in processing a dichotic tonal sequence. Journal of Experimental Psychology: Human Perception \& Performance, 2, 23-29.

HuAnG, W., Pastore, R. E., \& Hall, M. D. (1992). Octave illusion revisited [Abstract]. Journal of the Acoustical Society of America, 91, 2374.

REPP, B. H. (2001). Phase correction, phase resetting, and phase shifts after subliminal timing perturbations in sensorimotor synchronization. Journal of Experimental Psychology: Human Perception \& Performance, 27, 600-621.

Ross, J., Tervaniemi, M., \& NäÄtÄnen, R. (1996). Neural mechanisms of the octave illusion: Electrophysiological evidence for central origin. NeuroReport, 8, 303-306.

Sonnadara, R. R., \& Trainor, L. J. (2004). Perceived intensity effects in the octave illusion. Manuscript submitted for publication.

Terhardt, E. (1974). Pitch, consonance and harmony. Journal of the Acoustical Society of America, 55, 1061-1069.

ZWICKER, T. (1984). Experimente zür dichotischen Oktav-Tauschung. Acustica, 55, 128-136.

\section{NOTES}

1. Note that Deutsch (2004b) refers to the suppression model as the "two-channel" model.

2. Also of note are studies by Akerboom, ten Hoopen, and van der Knoop (1985) and Deutsch and Roll (1976), both of which reported no influence of silent intervals on perception of the illusion.

3. Deutsch (2004b) suggests that, according to Zwicker's (1984) findings, no illusion should have been obtained in some of the temporal conditions employed by Chambers et al. (2002). Note that according to this argument, no illusion should have been obtained in Experiment 3 of Deutsch (1980a), yet the illusion was clearly evident. This example highlights the need for caution in drawing conclusions from subjective report data.

4. In this experiment, listeners were presented with an octave illusion sequence and responded as rapidly as possible to a randomly oc- 
curring deviant stimulus. The results indicated that a $400-\mathrm{Hz}$ deviant was always more difficult to discriminate from the sequence than an $800-\mathrm{Hz}$ deviant, regardless of whether the $400-\mathrm{Hz}$ deviant replaced the high or the low pitch of the illusion.

5. Deutsch (2004b) also describes in detail a rebuttal to the claim that higher judgment variability in one condition of Deutsch (1980a) than in another could explain the pattern of results. However, Deutsch (2004b) has misunderstood the arguments against subjective measures proposed in Chambers et al. (2002). We noted that, when plotted as the percentage of subjective reports following the nondominant ear, the results in the standard octave illusion conditions of Deutsch (1980a, Condition 1) were usually more consistent than the results in the various control conditions (Condition 2). Deutsch (2004b) plots the data to show that when considered according to melodic contour or the location of the high-frequency component, the results in Condition 2 were highly consistent. We have no disagreement with this contention but emphasize that it is irrelevant to our criticism of the two-trial subjective report technique. Irrespective of whether the results in Condition 2 are replotted against a different factor to show reduced variability, subjective reports following the nondominant ear were more consistent in the standard octave illusion sequences (Condition 1) than in the various control sequences (Condition 2).

(Manuscript received December 18, 2003; revision accepted for publication April 30, 2004.) 